\title{
The semantic view of theories and higher-order languages
}

\author{
Laurenz Hudetz ${ }^{1}$ (D)
}

Received: 26 November 2016 / Accepted: 13 July 2017 / Published online: 31 October 2017

(C) The Author(s) 2017. This article is an open access publication

\begin{abstract}
Several philosophers of science construe models of scientific theories as set-theoretic structures. Some of them moreover claim that models should not be construed as structures in the sense of model theory because the latter are languagedependent. I argue that if we are ready to construe models as set-theoretic structures (strict semantic view), we could equally well construe them as model-theoretic structures of higher-order logic (liberal semantic view). I show that every family of set-theoretic structures has an associated language of higher-order logic and an up to signature isomorphism unique model-theoretic counterpart, which is able to serve the same purposes. This allows to carry over every syntactic criterion of equivalence for theories in the sense of the liberal semantic view to theories in the sense of the strict semantic view. Taken together, these results suggest that the recent dispute about the semantic view and its relation to the syntactic view can be resolved.
\end{abstract}

Keywords Structure of scientific theories - Semantic view of theories · Syntactic view of theories · Higher-order logic · Set-theoretic structures · Bourbaki · Equivalence of theories

This research was supported by a Marietta Blau Grant (ICM-2015-03060) sponsored by the Austrian Federal Ministry of Science and Research (BMWF).

$\bowtie \quad$ Laurenz Hudetz

laurenz.hudetz@sbg.ac.at

1 Department of Philosophy, University of Salzburg, Franziskanergasse 1, 5020 Salzburg, Austria 


\section{Introduction}

According to the syntactic view of scientific theories, we have to specify a class of statements in a formal language in order to present the formalism of a theory. According to the semantic view, in contrast, we have to specify a class of mathematical models to present the formalism of a theory. The recent debate about the syntactic and semantic view (Halvorson 2012, 2013; Glymour 2013; van Fraassen 2014; Lutz 2015) has shown that it is necessary to distinguish two ways in which the term 'mathematical model' can be understood in this context. It can mean (a) what is called 'structure' or 'model' in model theory, namely interpretations of a language of predicate logic or (b) set-theoretic structures consisting of a collection of basic sets together with a collection of set-theoretic constructs over those basic sets (e.g. relations, functions, functions of functions etc.).

Here is a simple example to illustrate this distinction. Suppose $\mathcal{A}=(P, L, I)$ is an affine plane in the sense of incidence geometry. The elements of $P$ are called 'points', those of $L$ 'lines' and $I$ is the incidence relation of $\mathcal{A}$. Now let $\left\{\sigma_{p}, \sigma_{l}, \epsilon\right\}$ be a signature (i.e. a set of symbols) providing a language for a synthetic axiomatisation of affine plane geometry and let $\tilde{\mathcal{A}}$ be an interpretation of this signature that maps the sort symbols for points and lines, $\sigma_{p}$ and $\sigma_{l}$, to the sets $P$ and $L$, respectively, and the incidence predicate $\epsilon$ to the set $I$. Then $\mathcal{A}$ is a set-theoretic structure, while $\tilde{\mathcal{A}}$ is a structure in the sense of model theory.

\begin{tabular}{cc}
$\mathcal{A}$ & $\tilde{\mathcal{A}}$ \\
\hline$(P, L, I)$ & $(P, L, I)$ \\
& $\uparrow \uparrow \uparrow$ \\
& $\left\{\sigma_{p}, \sigma_{l}, \epsilon\right\}$
\end{tabular}

This leads to a distinction between two versions of the semantic view. The liberal semantic view takes models of theories to be interpretations of formal languages ('model-theoretic structures'), whereas the strict semantic view takes them to be settheoretic structures. The difference between these two versions of the semantic view lies in their stances towards language independence. The liberal view embraces linguistic items (symbols) as parts of models whereas the strict view does not. To highlight this difference, Halvorson (2013) calls the liberal semantic view 'semantic $+\mathrm{L}$ ' and the strict semantic view 'semantic-L'.

Both versions of the semantic view have been criticised. Here is a brief summary of the main arguments put forward in the recent debate.

Some proponents of the semantic view reject its liberal version because they believe that we lose some of the benefits of the semantic view if we take models to be interpretations of formal languages. More specifically, the concern is that if models are interpretations of formal languages, then "the celebrated claim of the linguistic independence of considering models (and not first-order formalizations of theories), stressed by adherents of the semantic approach as giving it a clear advantage over the syntactic view, is simply not true" (French and Ladyman 1999, p. 114). An often-cited expression of this view is van Fraassen's (1989, p. 366) claim that the "impact of Suppes' innovation [i.e. presenting theories as classes of models] is lost if models are 
defined, as in many standard logic texts, to be partially linguistic entities, each yoked to a particular syntax."

In contrast, Halvorson (2012) argues that the (strict) semantic view is untenable. His strongest argument is that it lacks resources for explicating an adequate criterion of theoretical equivalence. Call this the problem of equivalence.

Glymour (2013) proposes a solution to the problem of equivalence. He points out that there are in fact good criteria of equivalence that can be applied to classes of modeltheoretic structures: e.g. definitional equivalence in the sense of Tarski et al. (1953) and synonymy in the sense of De Bouvere (1965). However, Glymour's proposal only protects the liberal semantic view and not the strict semantic view since it rests on the assumption that models are model-theoretic structures.

Halvorson (2013) takes Glymour's point but stresses that the liberal semantic view is not a real alternative to the syntactic view and that his criticism was aimed at the strict semantic view. Several other authors have also come to the conclusion that the liberal semantic view and a liberal version of the syntactic view should not be seen as rivals, e.g. Worrall (1984), Hendry and Psillos (2007) and Lutz (2014b, 2015). Glymour (2013) also makes this point. The liberal syntactic and semantic view both acknowledge that the formalism of a theory comprises a class of model-theoretic structures as well as a class of formulas. ${ }^{1}$ What distinguishes them is only what they focus on or what they regard as primary. The liberal syntactic view is what contemporary proponents of the syntactic view such as Lutz (2014a,b) endorse. Przełecki (1969) provides a systematic exposition of the liberal syntactic view, making use of models extensively. Several philosophers are happy with the liberal versions of both the syntactic and the semantic view. To them it is a pragmatic question whether to focus on formulas or model-theoretic structures satisfying these formulas in a given context—both are at our disposal.

van Fraassen (2014) puts forward two objections against Halvorson's criticism. (a) He contends that Halvorson misrepresents the semantic view. Van Fraassen points out that he has always acknowledged the trivial point that "when a theory is presented by displaying a class of models, those models are being described, and that description is something done in language" (van Fraassen 2014, p. 279). Furthermore, he underlines that, in his early Beth semantics approach, he explicitly included so-called elementary statements when reconstructing physical theories. Elementary statements are of the form 'magnitude $m$ has value $r$ at time $t$ '. Most equations and laws are therefore not elementary statements. (b) The second objection is that Halvorson ignores that models are representations and not merely uninterpreted mathematical structures. Van Fraassen points out that the same set-theoretic structures can be used to represent different phenomena and concludes that it is therefore no wonder that the problem of equivalence cannot be solved when ignoring representational aspects.

Lutz (2015, Sect. 6) analyses van Fraassen's reply to Halvorson and shows that it misses the point. (a) He argues that Halvorson's point goes beyond the trivial fact that

1 If we specify a class of sentences, we immediately get the class of all model-theoretic structures satisfying those sentences. If we specify a class of model-theoretic structures, we immediately get the class of all sentences that are satisfied in these structures. 
one has to use language to externally characterise ${ }^{2}$ a class of models and also beyond admitting elementary statements (which van Fraassen later abandoned in favour of an entirely language-free approach). ${ }^{3}$ The point is rather that a reasonable version of the semantic view has to construe models as model-theoretic structures of a formal object language in which the theory's specific formulas can be expressed. Van Fraassen has not conceded that point in his reply nor has he properly addressed it. (b) Lutz furthermore argues that Halvorson's counterexamples are even relevant when representation is taken into account because van Fraassen's account of representation and empirical adequacy is all about embeddability of data models, which is a purely formal relation preserved under isomorphism.

Lutz' latter point aside, it is important to note that Halvorson's concerns are simply not about representation at all. They are about how to present the formalism of a theory. The question is which kind of entities models should be taken to be when we reconstruct the formalism of a theory in philosophy of science (e.g. in order to provide a rigorous account of what it is to empirically and ontologically interpret a formalism or in order to define intertheoretic relations). Hence, the objection that Halvorson ignores representational aspects misses the point.

So the upshot of the debate so far is this: (1) There is wide agreement that the liberal semantic view and the liberal syntactic view are not real alternatives that we have to decide between. (2) There is unsettled disagreement regarding the liberal and the strict semantic view. The aim of this paper is to thoroughly address this disagreement.

\section{Higher-order model theory as peacemaker}

In what follows, I argue that if we are ready to construe models as set-theoretic structures (strict semantic view), we should also be ready to construe them as higherorder structures in the sense of model theory (liberal semantic and syntactic view). More specifically, I defend the following claims. (C1) All families of set-theoretic structures naturally give rise to internal languages of higher-order logic and corresponding families of model-theoretic structures of higher-order logic. The latter are exact counterparts of the former and able to serve the same purposes. (C2) Using internal languages, every solution to the problem of equivalence for classes of modeltheoretic structures can be carried over to classes of set-theoretic structures-pace Halvorson. (C3) Taking models to be model-theoretic structures of higher-order logic instead of set-theoretic structures does not lead to disadvantages-pace van Fraassen.

\footnotetext{
2 The language in which one externally characterises a class of models is not an object language that is interpreted by the models but rather what we could call an 'external metalanguage'. For example, the first-order language over the signature $\left\{\sigma_{p}, \sigma_{l}, \epsilon\right\}$ is the internal object language of the model-theoretic structure $\tilde{\mathcal{A}}$ described above, whereas the expression ' $\mathcal{A}=(P, L, I)$ is an affine plane' above belongs to the external metalanguage.

3 In retrospect van Fraassen (1989, p. 365) writes: "I soon found it much more advantageous to concentrate on the propositions expressible by elementary statements, rather than on the statements themselves. This is how my emphasis changed progressively in my articles on logical aspects of quantum mechanics, from 1968 onward. At later points there is not even a bow in the direction of syntactic description."
} 
These points are especially relevant for proponents of the strict semantic view, who can be divided into two groups: (1) There are those who construe models of scientific theories as set-theoretic structures without claiming that one could not equally well construe models as model-theoretic structures. For this group the results of this paper do not lead to a believe revision but rather to an update that the alternative is indeed equally viable. (2) There are those who go further, claiming that we cannot equally well construe models as model-theoretic structures because that would lead to a loss of certain advantages of the semantic view. The results of this paper refute that position.

Before I go into details, let me point out how my arguments are different and novel in comparison to the recent literature on the topic. Lutz (2015, p. 15) has made a similar point by arguing that there is no relevant difference between what he calls 'labelled structures' and 'indexed structures'. A labelled structure consists of a domain together with a mapping from a relational first-order signature to a set of first-order relations on the domain. So labelled structures are simply interpretations of singlesorted, relational first-order signatures. An indexed structure consists of a domain together with a mapping from an index set to a set of first-order relations on the domain. According to Lutz, the difference between labelled and indexed structures boils down to calling certain sets 'signatures' rather than 'index sets' —a merely verbal difference. One concern about this point is that it is either not strictly true or trivial. First, there seems to be a difference between signatures and mere index sets that needs to be addressed. Signatures associate types (arities) with their symbols, whereas mere index sets do not associate types with their elements. So more needs to be said in order to show that there is no substantial difference between indexed and labelled structures. Second, if one assumes that index sets do associate types with their elements from the start, then index sets in fact are signatures and indexed structures simply are labelled structures. But then the view that models are (set-theoretic) indexed structures rather than (model-theoretic) labelled structures would be clearly inconsistent from the start, which suggests that the reconstruction could not be charitable. Another concern is that labelled structures and indexed structures as described by Lutz are first-order structures. Assuming that all models are first-order oversimplifies the strict semanticists' account of models and, thus, threatens Lutz' point. As Halvorson (2012, p. 189) points out:

Granted, for a structure such as $\left\langle A, R_{1}, \ldots R_{n-1}\right\rangle$ [i.e. an indexed structure in Lutz' terminology] we can easily find a language $L$ for which it is an $L$ structure. But there are more complicated cases of mathematical structures-such as topological spaces - that cannot be derived in this way from a first-order language.

In fact, scientific practice relies heavily on more complicated set-theoretic structures. Proponents of the strict semantic view have no reason to assume that all models are of the simplest kind of relational first-order structures that Lutz discusses. On the contrary: the mathematical toolbox of the natural and social sciences is full with settheoretic structures that are not first-order. Topological spaces are just one simple example. Here are a few more: measure spaces including probability spaces (which play a role wherever stochastics is used), topological vector spaces (taking centre stage in functional analysis, which is vital to many areas of physics and, through the work of von Neumann (1937), also to economics), differentiable manifolds (which 
are crucial in mechanics and spacetime physics), stochastic processes (used to model stock market exchange and weather processes), games in the sense of game theory (modelling social conflict and cooperation) and so on and so forth.

So it is necessary to generalise Lutz' point. Instead of showing that there is no substantial difference between indexed and labelled first-order structures, we need to establish that every family of set-theoretic structures-no matter how complex the structures are-has a model-theoretic counterpart that is able to serve the same purposes.

The crucial question is whether that is possible at all. My point is that it is indeed possible given that we use higher-order predicate logic of order $\omega$ (short: 'HOL') as the logical framework. This liberality in the choice of logic enables us to find internal languages for arbitrary families of set-theoretic structures only by determining how they are constructed. Moreover, this choice of logic cannot be regarded as too liberal since even the syntactic view of theories embraces HOL. For example, Carnap (1958) explicitly took languages of scientific theories to be HOL-languages.

\section{Higher-order languages for set-theoretic structures}

In order to show that all families of set-theoretic structures have naturally corresponding families of HOL-structures as their model-theoretic counterparts (Sect. 3.3), we first have to make the concept of set-theoretic structure precise (Sect. 3.1) and describe HOL-languages and HOL-structures (Sect. 3.2).

\subsection{Set-theoretic structures}

Following Suppes, many proponents of the semantic view have-either explicitly or implicitly_assumed that models are mathematical structures consisting of a collection of basic sets together with a collection of set-theoretic constructs over those basic sets. The definition below captures this notion of structure in its most general form.

Definition $1(\mathcal{B}, \mathcal{C})$ is a set-theoretic structure iff

(1) $\mathcal{B}$ is a non-empty sequence of non-empty sets (called 'basic sets') and

(2) $\mathcal{C}$ is a countable ordinal-indexed sequence where every member is either an element of some basic set in $\mathcal{B}$ or a set-theoretic construct over the basic sets $\mathcal{B}$.

There are no further constraints regarding basic sets. They may have abstract objects as members, but concrete entities are equally admissible. Set-theoretic constructs over basic sets are what can be obtained by (repeatedly) taking Cartesian products and power sets. Starting from basic sets, we can for instance easily obtain relations, functions, relations between relations, functions of functions (such as operators or functionals in the sense of functional analysis) and so on.

All or nearly all mathematical structures that are used as models in the sciences can be viewed as set-theoretic structures as defined above. This also includes state space structures in the sense of van Fraassen (1989). However, in order to rigorously show that the definition of 'set-theoretic structure' applies in a given case, the notion 
of set-theoretic construct needs to be made more precise. The most well-known way of doing this goes back to Bourbaki (1968, pp. 259-260) and rests on their notion of echelon construction schemes. As Halvorson (2012, p. 189) points out, it has been explicitly adopted by various philosophers of science advocating the semantic view. For instance, Da Costa and French (2003), the Munich school of structuralists (Balzer et al. 1987) or Scheibe (1997, Chapter II) use Bourbaki's explication, which can be reconstructed as follows.

\section{Definition 2}

(a) $\left(s_{1}, \ldots, s_{n}\right)$ is an echelon construction scheme for $\kappa$ basic sets iff $\kappa$ is an ordinal less than or equal to $\omega$ and for every $k \in \mathbb{N}$ with $1 \leq k \leq n$ : $s_{k}$ is a pair of natural numbers such that

(1) if $\pi_{1}\left(s_{k}\right)=0$ and $\kappa<\omega$, then $1 \leq \pi_{2}\left(s_{k}\right) \leq \kappa$.

(2) if $\pi_{2}\left(s_{k}\right)=0$, then $1 \leq \pi_{1}\left(s_{k}\right) \leq k-1$.

(3) if $\pi_{1}\left(s_{k}\right) \neq 0 \neq \pi_{2}\left(s_{k}\right)$, then $1 \leq \pi_{1}\left(s_{k}\right) \leq k-1$ and $1 \leq \pi_{2}\left(s_{k}\right) \leq k-1$.

(Here, $\pi_{1}$ and $\pi_{2}$ are the canonical projections for ordered pairs.)

(b) $\left(E_{1}, \ldots, E_{n}\right)$ is the echelon construction over basic sets $\mathcal{B}$ given by construction scheme $\left(s_{1}, \ldots, s_{n}\right)$ iff $\left(s_{1}, \ldots, s_{n}\right)$ is an echelon construction scheme for $|\mathcal{B}|$ basic sets and for all $k$ with $1 \leq k \leq n$ :

(1) if $\pi_{1}\left(s_{k}\right)=0$, then $E_{k}=\mathcal{B}_{\pi_{2}\left(s_{k}\right)}$, where $\mathcal{B}_{\pi_{2}\left(s_{k}\right)}$ is the $\pi_{2}\left(s_{k}\right)$-th basic set.

(2) if $\pi_{2}\left(s_{k}\right)=0$, then $E_{k}=\mathcal{P}\left(E_{\pi_{1}\left(s_{k}\right)}\right)$.

(3) if $\pi_{1}\left(s_{k}\right) \neq 0 \neq \pi_{2}\left(s_{k}\right)$, then $E_{k}=E_{\pi_{1}\left(s_{k}\right)} \times E_{\pi_{2}\left(s_{k}\right)}$.

(c) $C$ is a set-theoretic construct over basic sets $\mathcal{B}$ iff $C$ is a subset of the last component of an echelon construction over basic sets $\mathcal{B}$ given by some echelon construction scheme.

Let us take a simple example to see how these definitions work. We show that every topological group $(X, *, \mathcal{T})$ is a set-theoretic structure with a sequence of basic sets having only one member $(X)$ and a two-element sequence $(*, \mathcal{T})$ of set-theoretic constructs over $X$. To see that the group operation $*$ and the topology $\mathcal{T}$ are settheoretic constructs over $X$, use the definitions above and note that the following statements hold.

(1) $S_{1}=((0,1),(1,1),(2,1))$ and $S_{2}=((0,1),(1,0))$ are echelon construction schemes for one basic set.

(2) $(X, X \times X,(X \times X) \times X)$ and $(X, \mathcal{P}(X))$ are the echelon constructions over $X$ given by the construction schemes $S_{1}$ and $S_{2}$, respectively.

(3) $* \subseteq(X \times X) \times X$ and $\mathcal{T} \subseteq \mathcal{P}(X)$.

Similarly, one can show that measure spaces, topological vector spaces, differentiable manifolds, stochastic processes, all kinds of dynamical systems or games in the sense of game theory are set-theoretic structures using Bourbaki's definitions. ${ }^{4}$

\footnotetext{
4 However, in practice one never checks whether Bourbaki's definitions are satisfied when specifying set-theoretic structures. The definitions of 'echelon construction scheme' and 'echelon construction' are awkward to use and there is no reward in unpacking them each time. Once one knows how they work, it is clear when and that they apply. Their point is not that we need them in practice but rather to provide-for theoretical purposes - a mathematically rigorous account of the formal architecture all kinds of set-theoretic structures that we find in practice share.
} 
Set-theoretic structures come in different kinds. The models of a theory are usually of one kind in the sense that they are set-theoretically constructed in the same way. Definition 3 below explicates what kinds of set-theoretic structures are and what it means that different set-theoretic structures are of the same kind. But first we need two auxiliary definitions. Since different echelon construction schemes can lead to the same construction result, it is useful to define an equivalence relation for echelon construction schemes: $S \equiv S^{\prime}$ iff $S$ and $S^{\prime}$ are both echelon construction schemes for $\kappa$ basic sets and, given a sequence of $\kappa$ basic sets $\mathcal{B}$, the final component of the echelon construction over $\mathcal{B}$ given by $S$ is identical to the final component of the echelon construction over $\mathcal{B}$ given by $S^{\prime}$. Moreover, let us use the notation 'index $(\mathcal{X})$ ' for the ordinal which serves as the index set of a given ordinal-indexed sequence $\mathcal{X}$.

\section{Definition 3}

(a) $\mathfrak{K}$ is a kind of set-theoretic structures iff $\mathfrak{K}$ consists of

(1) an ordinal $\kappa$ such that $1 \leq \kappa \leq \omega$ together with

(2) a countable ordinal-indexed sequence $\mathfrak{S}$ such that for every $\alpha \in$ index (S), $\mathfrak{S}_{\alpha}$ is either an equivalence class of echelon construction schemes for $\kappa$ basic sets or a natural number less than or equal to $\kappa$.

(b) A set-theoretic structure $(\mathcal{B}, \mathcal{C})$ is of kind $\mathfrak{K}=(\kappa, \mathfrak{S})$ iff the sequence $\mathcal{B}$ is of length $\kappa$ and index $(\mathcal{C})=$ index $(\mathfrak{S})$ and for every $\alpha \in$ index $(\mathfrak{S})$ :

(1) if $\mathfrak{S}_{\alpha}$ is a natural number, then $\mathcal{C}_{\alpha}$ is an element of the basic set $\mathcal{B}_{\mathfrak{S}_{\alpha}}$.

(2) if $\mathfrak{S}_{\alpha}$ is an equivalence class of echelon construction schemes, then $\mathcal{C}_{\alpha}$ is a set-theoretic construct over $\mathcal{B}$ given by the construction schemes in $\mathfrak{S}_{\alpha}$.

(c) $\mathbf{C}$ is a class of set-theoretic structures of the same kind (shorter: family of settheoretic structures) iff $\mathbf{C}$ is a class of set-theoretic structures and there is exactly one kind of set-theoretic structures, $\mathfrak{K}$, such that every set-theoretic structure in $\mathbf{C}$ is of kind $\mathfrak{K}^{5}$

So much for set-theoretic structures. The next section introduces the syntax of higherorder logic as well as higher-order structures.

\subsection{Structures of higher-order logic}

There are many ways to specify a syntax for higher-order logic. Probably the most prominent syntax is that of Church's simple type theory, which is based on function symbols only and allows to quantify over propositions. However, for our purposes it is more convenient to use a syntax that is closer to first-order predicate logic (cf. Orey 1959). Our higher-order languages have predicate variables and predicate constants of all orders as well as the usual logical and auxiliary symbols. In contrast, first-order languages have only predicate constants of order 1 and no predicate variables.

\footnotetext{
5 Being of the same kind rules out ambiguous cases where the kind of set-theoretic structures is not uniquely determined by their set-theoretic construction. For example, the singleton containing only the structure $(\mathbb{R}, \emptyset)$ is ambiguous in this respect. But as soon as we add another structure with a non-empty construct to this class, its kind of structures becomes uniquely determined.
} 
We have to assign types (arities) to predicate constants and variables. We use the type constructors $\otimes$ and $\wp$ to generate types from basic types in the following way (cf. Lambek and Scott 1986; Awodey 1997; Johnstone 2002).

Definition 4 The full type hierarchy types $(B)$ over a non-empty set $B$ of basic type symbols is defined as follows:

(1) Every basic type symbol of $B$ is in types $(B)$.

(2) If $\tau_{1}, \tau_{2}$ are in types $(B)$, then $\tau_{1} \otimes \tau_{2}$ is in types $(B)$. ('product types')

(3) If $\tau$ is in types $(B)$, then $\wp(\tau)$ is in types $(B)$. ('power types')

For brevity's sake, I will also say 'type' instead of 'type symbol'. Basic types and power types are called 'simple types'. HOL-signatures (vocabularies) can be defined as follows.

\section{Definition 5 A HOL-signature $\Sigma$ consists of}

(1) a set of basic type symbols,

(2) a set of constant symbols and

(3) a mapping associating with each constant a simple type over the basic types.

Constant symbols of basic types are called 'individual constants' and constant symbols of power types are called 'predicate constants'. Given a HOL-signature $\Sigma$, formulas and sentences of $\Sigma$ are defined as usual (Awodey 1997; Johnstone 2002). The resulting higher-order language over $\Sigma$ is denoted by ' $L_{\omega}(\Sigma)$ '.

In the next step, we introduce HOL-structures, i.e. interpretations of HOLsignatures. For our purposes it suffices to consider only standard HOL-structures as opposed to general structures (cf. Henkin 1950). This allows to slightly simplify the usual definition, which Orey (1959, pp. 73-74) spells out in detail.

Definition 6 Let $\Sigma$ be a HOL-signature.

(a) $\mathcal{B}$ is a collection of basic sets for $\Sigma$ iff $\mathcal{B}$ assigns non-empty sets to the basic types of $\Sigma$.

(b) Given a collection $\mathcal{B}$ of basic sets for $\Sigma$, the expansion $\mathcal{B}^{+}$of $\mathcal{B}$ to the full type hierarchy over the basic types of $\Sigma$ is defined as follows:

(1) $\mathcal{B}^{+}(\beta)=\mathcal{B}(\beta)$ if $\beta$ is a basic type of $\Sigma$.

(2) $\mathcal{B}^{+}\left(\tau_{1} \otimes \tau_{2}\right)=\mathcal{B}^{+}\left(\tau_{1}\right) \times \mathcal{B}^{+}\left(\tau_{2}\right)$ if $\tau_{1}, \tau_{2}$ are types of $\Sigma$.

(3) $\mathcal{B}^{+}(\wp(\tau))=\mathcal{P}\left(\mathcal{B}^{+}(\tau)\right)$ if $\tau$ is a type of $\Sigma$.

(c) $(\mathcal{B}, \mathcal{C})$ is a $\Sigma$-structure iff

(1) $\mathcal{B}$ is a collection of basic sets for $\Sigma$ and

(2) $\mathcal{C}$ maps every $\Sigma$-constant $c$ of type $\tau$ to an element of $\mathcal{B}^{+}(\tau)$.

Satisfaction of a formula $\varphi$ of $L_{\omega}(\Sigma)$ in a $\Sigma$-structure $\mathcal{A}$ by a sequence of objects $\bar{a}$ (short: $\mathcal{A} \vDash \varphi[\bar{a}])$ and truth $(\mathcal{A} \vDash \varphi)$ can be defined as usual.

Example One can view topological spaces as HOL-structures. First, one needs a suitable HOL-signature $\Sigma_{\text {TOP }}$ consisting of a basic type symbol $\sigma$ and a constant symbol $T$ of type $\wp \wp(\sigma)$, standing for the point set and the topology on it, respectively. A

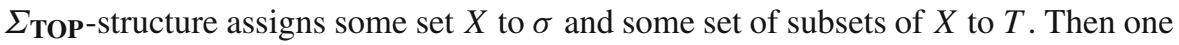
can characterise various kinds of topological spaces qua $\Sigma_{\mathbf{T O P}}$-structures by means of 
the sentences they satisfy. For instance, Hausdorff spaces are those $\Sigma_{\mathbf{T O P}}$-structures that satisfy $(\models)$ the following sentences of $L_{\omega}\left(\Sigma_{\text {TOP }}\right)$ :

$$
\begin{gathered}
\emptyset \in T \wedge \sigma \in T \\
\forall_{A, B \in \wp(\sigma)} A \cap B \in T \\
\forall_{F \in \wp(T)} \bigcup F \in T \\
\forall_{x, y \in \sigma}\left(x \neq y \rightarrow \exists_{A, B \in T}(x \in A \wedge y \in B \wedge A \cap B=\emptyset)\right)
\end{gathered}
$$

The names of these formulas are written using metalinguistic abbreviation conventions for HOL-formulas (cf. Awodey 1997, p. 4).

\subsection{Model-theoretic counterparts of set-theoretic structures}

This section is devoted to showing that, given a family of set-theoretic structures of the same kind, there is a suitable higher-order signature that fits these structures. This allows us to transform set-theoretic structures into corresponding HOL-structures.

Suppose $\mathbf{C}$ is a family of set-theoretic structures. Then there is exactly one kind of set-theoretic structures $\mathfrak{K}=(\kappa, \mathfrak{S})$ such that every structure in $\mathbf{C}$ is of kind $\mathfrak{K}$. With this at hand, we define a signature. We use natural numbers as basic types $B T_{\mathbf{C}}:=\left\{\beta_{i}: i \in \mathbb{N} \& i \leq \kappa\right\}$ with $\beta_{i}:=i$. Let $\alpha \in \operatorname{index}(\mathfrak{S})$. If $\mathfrak{S}_{\alpha}$ is an equivalence class of echelon construction schemes in $\mathfrak{S}$, we specify for every echelon construction scheme $\left(s_{1}, \ldots, s_{n}\right)$ in $\mathfrak{S}_{\alpha}$ a sequence $\operatorname{aux}\left(s_{1}, \ldots, s_{n}\right):=\left(\tau_{1}, \ldots, \tau_{n}\right)$ of types from the full type hierarchy types $\left(B_{\mathbf{C}}\right)$ such that for all $k$ with $1 \leq k \leq n$ :

(1) if $\pi_{1}\left(s_{k}\right)=0$, then $\tau_{k}$ is the basic type $\beta_{\pi_{2}\left(s_{k}\right)}$.

(2) if $\pi_{2}\left(s_{k}\right)=0$, then $\tau_{k}$ is the power type $\wp\left(\tau_{\pi_{1}\left(s_{k}\right)}\right)$.

(3) if $\pi_{1}\left(s_{k}\right) \neq 0 \neq \pi_{2}\left(s_{k}\right)$, then $\tau_{k}$ is the product type $\tau_{\pi_{1}\left(s_{k}\right)} \otimes \tau_{\pi_{2}\left(s_{k}\right)}$.

The power type of the last member of aux $\left(s_{1}, \ldots, s_{n}\right)$, i.e. $\wp\left(\tau_{n}\right)$, is called 'the type corresponding to the echelon construction scheme $\left(s_{1}, \ldots, s_{n}\right)$ '. Within one equivalence class, all echelon construction schemes have the same corresponding type. This justifies to speak of 'the type corresponding to the equivalence class $\mathfrak{S}_{\alpha}$ '. Using these preliminaries, we can define the HOL-signature corresponding to a given kind of set-theoretic structures.

Definition 7 Let $\mathbf{C}$ be a family of set-theoretic structures and let $\mathfrak{K}=(\kappa, \mathfrak{S})$ be its kind. We define the canonical HOL-signature $\Sigma_{\mathbf{C}}$ associated with $\mathbf{C}$ as follows:

(1) The basic types of $\Sigma_{\mathbf{C}}$ are given by $B T_{\mathbf{C}}$.

(2) The constant symbols of $\Sigma_{\mathbf{C}}$ are given by $C S_{\mathbf{C}}:=\left\{c_{\alpha}: \alpha \in\right.$ index (S) $\}$, where $c_{\alpha}:=\left(\alpha, \mathfrak{S}_{\alpha}\right)$.

(3) The constants of $\Sigma_{\mathbf{C}}$ are mapped to types as follows. For every $\alpha \in \operatorname{index}(\mathfrak{S})$ : if $\mathfrak{S}_{\alpha}$ is a natural number, then $c_{\alpha}$ is of type $\beta_{\mathfrak{S}_{\alpha}}$; if $\mathfrak{S}_{\alpha}$ is an equivalence class of echelon construction schemes, then $c_{\alpha}$ is of the type corresponding to the equivalence class $\mathfrak{S}_{\alpha}$. 
Note that the signature $\Sigma_{\mathbf{C}}$ depends only on the kind $\mathfrak{K}$ of $\mathbf{C}$. So distinct classes $\mathbf{C} \neq \mathbf{C}^{\prime}$ of set-theoretic structures of the same kind may have the same canonical signature $\Sigma_{\mathbf{C}}=\Sigma_{\mathbf{C}^{\prime}}$. For example, different classes of topological spaces (e.g. $T_{0}, \ldots, T_{6}$ ) all have the same canonical signature. The higher-order language $L_{\omega}\left(\Sigma_{\mathbf{C}}\right)$ over the signature $\Sigma_{\mathbf{C}}$ can be viewed as the internal language of the set-theoretic structures in the family $\mathbf{C}$.

Now we know how to construct a HOL-signature for a given family of set-theoretic structures. This raises the question how unique this signature is. More precisely, the question is whether there could be other HOL-signatures that fit the same class of settheoretic structures but are inequivalent to the canonical signature. In order to answer that question we have to clarify what the relevant notion of equivalence between signatures is and what it means that a signature fits a family of set-theoretic structures.

Two signatures are equivalent for all purposes just in case there is a signature isomorphism between them. Signature isomorphisms capture the idea of changing symbols while leaving everything else the same.

Definition 8 A HOL-signature isomorphism $h: \Sigma \rightarrow \Sigma^{\prime}$ consists of

(1) a bijection $h_{t y p}$ from the basic type symbols of $\Sigma$ to those of $\Sigma^{\prime}$ and

(2) a bijection $h_{c o n}$ from the constant symbols of $\Sigma$ to those of $\Sigma^{\prime}$ such that for all constant symbols $c$ of $\Sigma, c$ is of type $\tau$ in $\Sigma$ iff $h_{c o n}(c)$ is of type $h_{t y p}^{+}(\tau)$ in $\Sigma^{\prime}$ (where $h_{\text {typ }}^{+}$is the extension of $h_{\text {typ }}$ to the full type hierarchy commuting with the type constructors $\wp$ and $\otimes$ ).

The idea of signature morphisms is taken from and plays a central role in institution theory, which is a very general theory of systems consisting of syntax, models and a satisfaction relation (cf. Goguen and Burstall 1992; Diaconescu 2008). Institution theory generalises several concepts and results from first-order model theory.

A signature $\Sigma$ fits a family of set-theoretic structures if it bears a suitable relationship to the kind $\mathfrak{K}$ of these structures. The idea is that it or an equivalent signature could arise canonically from $\mathfrak{K}$. In order to make this more precise, we exploit the fact that the canonical signature $\Sigma_{\mathbf{C}}$ of a family $\mathbf{C}$ of set-theoretic structures faithfully encodes the kind $\mathfrak{K}$ of these structures.

Definition 9 A HOL-signature $\Sigma$ fits a family $\mathbf{C}$ of set-theoretic structures iff $\Sigma$ is isomorphic to the canonical signature $\Sigma_{\mathbf{C}}$ associated with $\mathbf{C}$.

This definition has immediate consequences regarding existence and uniqueness of HOL-signatures fitting a given class of set-theoretic structures.

\section{Lemma 1}

(a) If $\mathbf{C}$ is a family of set-theoretic structures, then its canonical signature $\Sigma_{\mathbf{C}}$ fits $\mathbf{C}$.

(b) If two HOL-signatures $\Sigma, \Sigma^{\prime}$ both fit the same family of set-theoretic structures, then $\Sigma$ is isomorphic to $\Sigma^{\prime}$.

So given a class $\mathbf{C}$ of set-theoretic structures of the same kind, the canonical signature $\Sigma_{\mathbf{C}}$ is up to signature isomorphism the only signature that fits $\mathbf{C}$.

There is a sense in which a model-theoretic structure can be virtually the same as a set-theoretic structure in the sense that it is able to serve the purposes. The example in 
the introduction illustrates this: an affine plane $(P, L, I)$ is virtually the same as the interpretation of the first-order signature $\left\{\sigma_{p}, \sigma_{l}, \epsilon\right\}$ of affine geometry that maps $\sigma_{p}$ to $P, \sigma_{l}$ to $L$ and $\epsilon$ to $I$. The only difference is that, when an affine plane is viewed as a set-theoretic structure, its components are ordered in a sequence, whereas they are named by symbols when viewed as a model-theoretic structure. In such cases of virtual sameness between a HOL-structure and a set-theoretic structure, it is apt to say that the former is a model-theoretic counterpart of the latter or that the former interprets its signature over the latter. The next definition makes this concept precise.

Definition 10 Suppose $\mathbf{C}$ is a family of set-theoretic structures and $\Sigma$ is a HOLsignature that fits $\mathbf{C}$ with signature isomorphism $h: \Sigma \rightarrow \Sigma_{\mathbf{C}}$. Moreover, suppose that $\mathcal{A}=(\mathcal{B}, \mathcal{C})$ is in $\mathbf{C}$ and that $\tilde{\mathcal{A}}=(\tilde{\mathcal{B}}, \tilde{\mathcal{C}})$ is a $\Sigma$-structure. Then we say that $\tilde{\mathcal{A}}$ is a $\Sigma$-counterpart of $\mathcal{A}$ w.r.t. $h$ iff

(a) for every basic type $\beta$ of $\Sigma, \tilde{\mathcal{B}}$ assigns the basic set $\mathcal{B}_{h(\beta)}$ to $\beta$;

(b) for every constant $c$ of $\Sigma, \tilde{\mathcal{C}}$ assigns the set-theoretic construct $\mathcal{C}_{\alpha}$ to $c$, where $\alpha$ is the first component of the pair $h(c)$.

It follows that $\Sigma$-counterparts exist and are uniquely determined. More precisely:

Lemma 2 Suppose $\mathbf{C}$ is a family of set-theoretic structures and $\Sigma$ is a HOL-signature that fits $\mathbf{C}$ with signature isomorphism $h: \Sigma \rightarrow \Sigma_{\mathbf{C}}$. If $\mathcal{A}$ is in $\mathbf{C}$, then there is exactly one $\Sigma$-structure $\tilde{\mathcal{A}}$ that is a $\Sigma$-counterpart of $\mathcal{A}$ w.r.t. $h$.

The next definition explains what it means that a class of HOL-structures is a modeltheoretic counterpart of a class of set-theoretic structures.

Definition 11 Suppose $\mathbf{C}$ is a family of set-theoretic structures and $\Sigma$ is a HOLsignature that fits $\mathbf{C}$ with signature isomorphism $h: \Sigma \rightarrow \Sigma_{\mathbf{C}}$. Let $\tilde{\mathbf{C}}$ be a class of $\Sigma$-structures. We say that $\tilde{\mathbf{C}}$ is a $\Sigma$-counterpart of $\mathbf{C}$ w.r.t. $h$ iff there is a unique bijection $F$ from $\mathbf{C}$ to $\tilde{\mathbf{C}}$ such that for all $\mathcal{A} \in \mathbf{C}, F(\mathcal{A})$ is a $\Sigma$-counterpart of $\mathcal{A}$ w.r.t. $h$.

With these definitions and lemmata at hand, we can prove the main result of this section. Loosely speaking, it says that given a family of models in the sense of the strict semantic view, we can transform it into a family of models in the sense of the liberal semantic view that is able to serve the same purposes.

Proposition For every family $\mathbf{C}$ of set-theoretic structures, there is up to signature isomorphism exactly one HOL-signature $\Sigma$ fitting $\mathbf{C}$ and exactly one class of $\Sigma$ structures $\tilde{\mathbf{C}}$ such that $\tilde{\mathbf{C}}$ is a $\Sigma$-counterpart of $\mathbf{C}$ (w.r.t. a given signature isomorphism).

Proof Let $\mathbf{C}$ be a family of set-theoretic structures. We know from Lemma 1 that there is up to signature isomorphism exactly one signature $\Sigma$ that fits $\mathbf{C}$. Let $\Sigma$ be a signature that fits $\mathbf{C}$ with signature isomorphism $h: \Sigma \rightarrow \Sigma_{\mathbf{C}}$. Then by Lemma 2, every $\mathcal{A} \in \mathbf{C}$ has exactly one $\Sigma$-counterpart w.r.t. $h$. Let $(\cdot)^{\Sigma, h}$ be the function that maps every $\mathcal{A} \in \mathbf{C}$ to its $\Sigma$-counterpart $\mathcal{A}^{\Sigma, h}$ w.r.t. $h$. Let $\tilde{\mathbf{C}}$ be the image of $(\cdot)^{\Sigma, h}$. Then $(\cdot)^{\Sigma, h}: \mathbf{C} \rightarrow \tilde{\mathbf{C}}$ is surjective by definition.

Furthermore, $(\cdot)^{\Sigma, h}: \mathbf{C} \rightarrow \tilde{\mathbf{C}}$ is injective. Let $\mathcal{A}=(\mathcal{B}, \mathcal{C})$ and $\mathcal{A}^{\prime}=\left(\mathcal{B}^{\prime}, \mathcal{C}^{\prime}\right)$ be in $\mathbf{C}$ and suppose $\mathcal{A}^{\Sigma, h}=\left(\mathcal{A}^{\prime}\right)^{\Sigma, h}$. This together with Definition 10 yields: (1) For every basic type $\beta$ of $\Sigma, \mathcal{B}_{h(\beta)}=\mathcal{B}^{\Sigma, h}(\beta)=\left(\mathcal{B}^{\prime}\right)^{\Sigma, h}(\beta)=\mathcal{B}_{h(\beta)}^{\prime}$. (2) For every 
constant $c$ of $\Sigma, \mathcal{C}_{\alpha}=\mathcal{C}^{\Sigma, h}(c)=\left(\mathcal{C}^{\prime}\right)^{\Sigma, h}(c)=\mathcal{C}_{\alpha}^{\prime}$, where $\alpha$ is the first component of the pair $h(c)$. Let $\mathfrak{K}=(\kappa, \mathfrak{S})$ be the kind of $\mathbf{C}$. Then $h: \Sigma \rightarrow \Sigma_{\mathbf{C}}$ being a signature isomorphism together with the above equations implies: (3) For $i \in \mathbb{N}$ with $i \leq \kappa, \mathcal{B}_{i}=\mathcal{B}^{\Sigma, h}\left(h^{-1}(i)\right)=\left(\mathcal{B}^{\prime}\right)^{\Sigma, h}\left(h^{-1}(i)\right)=\mathcal{B}_{i}^{\prime}$. (4) For every $\alpha \in \operatorname{index}(\mathfrak{S})$, $\mathcal{C}_{\alpha}=\mathcal{C}^{\Sigma, h}\left(h^{-1}\left(\left(\alpha, \mathfrak{S}_{\alpha}\right)\right)\right)=\left(\mathcal{C}^{\prime}\right)^{\Sigma, h}\left(h^{-1}\left(\left(\alpha, \mathfrak{S}_{\alpha}\right)\right)\right)=\mathcal{C}_{\alpha}^{\prime}$. Hence, $\mathcal{A}=\mathcal{A}^{\prime}$.

Thus, $(\cdot)^{\Sigma, h}: \mathbf{C} \rightarrow \tilde{\mathbf{C}}$ is a bijection that maps every $\mathcal{A} \in \mathbf{C}$ to its $\Sigma$-counterpart w.r.t. $h$. Moreover, every bijection that does this coincides with $(\cdot)^{\Sigma, h}$. So by Definition $11, \tilde{\mathbf{C}}$ is a $\Sigma$-counterpart of $\mathbf{C}$ w.r.t. $h$. It follows from Lemma 2 that $\tilde{\mathbf{C}}$ is the only $\Sigma$-counterpart of $\mathbf{C}$ w.r.t. $h$.

In essence, this proposition gives us a function $\mathbf{C} \mapsto \mathbf{C}^{\Sigma, h}$ that maps any given family of set-theoretic structures $\mathbf{C}$ to its unique $\Sigma$-counterpart $\mathbf{C}^{\Sigma, h}$ w.r.t. $h$. Note that the counterpart mapping has two parameters: $\Sigma$ and $h$. So one family of settheoretic structures has model-theoretic counterparts in various isomorphic signatures. Of course, all of these counterparts are indistinguishable apart from the choice of symbols in their signatures. Yet, it is sometimes useful to have a canonical counterpart at hand. For a set-theoretic structure $\mathcal{A} \in \mathbf{C}$, its canonical counterpart is defined as the $\Sigma_{\mathbf{C}}$-counterpart of $\mathcal{A}$ w.r.t. the identity-isomorphism on $\Sigma_{C}$. We denote the canonical counterpart of $\mathcal{A} \in \mathbf{C}$ by ' $\mathcal{A}^{\Sigma_{\mathbf{C}}}$ '. The canonical counterpart of the family $\mathbf{C}$ itself is given by $\mathbf{C}^{\Sigma_{\mathbf{C}}}=\left\{\mathcal{A}^{\Sigma_{\mathbf{C}}}: \mathcal{A} \in \mathbf{C}\right\}$.

Example We consider the class $\mathbf{H}$ of Hausdorff spaces qua set-theoretic structures in order to illustrate how the described method works. Proponents of the strict semantic view could specify this family of set-theoretic structures as follows: $(X, \mathcal{T})$ is a member of $\mathbf{H}$ iff $X$ is a non-empty set and $\mathcal{T} \subseteq \mathcal{P}(X)$ such that $\emptyset$ and $X$ are in $\mathcal{T}, \mathcal{T}$ is closed under binary intersections, $\mathcal{T}$ is closed under arbitrary unions and $(X, \mathcal{T})$ has the Hausdorff-property. (For brevity, I denote sequences with just one member by the name of the member itself if there is no danger of confusion.) As we already know from the example at the end of Sect. 3.2, $\mathcal{T}$ is a set-theoretic construct over $X$ given by the echelon construction scheme $S:=((0,1),(1,0))$. So every topological space $(X, \mathcal{T})$ is a structure of kind top $:=\left(1,[S]_{\equiv}\right)$. Now we construct the canonical HOLsignature for $\mathbf{H}$ using the method described above.

(1) First, note that there is only one basic type in $B T_{\mathbf{H}}$, namely 1.

(2) The type in types $\left(B T_{\mathbf{H}}\right)$ that corresponds to the equivalence class $[S]_{\equiv}$ is $\wp(\wp(1))$.

(3) The canonical signature $\Sigma_{\mathbf{H}}$ consists of the basic type 1 and the predicate constant $\left(1,[S]_{\equiv}\right)$ of type $\wp(\wp(1))$. This is up to signature isomorphism the only signature that fits $\mathbf{H}$. It is convenient to introduce simpler metalinguistic names for the symbols of $\Sigma_{\mathbf{H}}$. Let ' $\sigma$ ' denote the type symbol 1 and let ' $T$ ' denote the constant symbol $\left(1,[S]_{\equiv}\right)$.

(4) $L_{\omega}\left(\Sigma_{\mathbf{H}}\right)$ is the internal higher-order language of Hausdorff spaces.

For any Hausdorff space qua set-theoretic structure, $(X, \mathcal{T})$, its canonical counterpart $(X, \mathcal{T})^{\Sigma_{\mathbf{H}}}$ assigns the basic set $X$ to the basic type symbol $\sigma$ and the set of sets $\mathcal{T}$ to the constant symbol $T$. Of course, the canonical counterpart of a Hausdorff space qua set-theoretic structure should turn out to be a Hausdorff spaces qua $\Sigma_{\mathbf{H}}$-structures, 
meaning that it should satisfy the characteristic $L_{\omega}\left(\Sigma_{\mathbf{H}}\right)$-axioms listed in the example at the end of Sect. 3.2. One can show that this is indeed the case.

\section{Corollary}

(a) $(X, \mathcal{T})$ is a Hausdorff space qua set-theoretic structure iff its canonical counterpart $(X, \mathcal{T})^{\Sigma_{\mathbf{H}}}$ is a Hausdorff space qua $\Sigma_{\mathbf{H}}$-structures.

(b) The canonical counterpart $\mathbf{H}^{\Sigma_{\mathbf{H}}}$ of $\mathbf{H}$ coincides with the class $\mathbf{H}_{\mathrm{hol}}$ of Hausdorff spaces qua $\Sigma_{\mathbf{H}}$-structures.

Granted, this is just a toy example. Nonetheless, it shows how Halvorson's (2012, p. 189) worry that "there are more complicated cases of mathematical structures - such as topological spaces - that cannot be derived in this way from a first-order language" can be met by the proposed method of constructing internal higher-order signatures and languages. Moreover, we know from the proposition above that this method works for all set-theoretic structures-no matter how complex they are. For example, it is not hard to specify a HOL-signature for smooth manifolds qua model-theoretic structures. Future work will examine more elaborate examples like that in detail.

\section{Consequences for the syntax-semantics debate}

The conclusion of the last section is that every family of models in the sense of the strict semantic view has a model-theoretic counterpart: a family of models in the sense of the liberal semantic view. There is a sense in which families of set-theoretic structures encode syntactic information that can be turned into a full-fledged "yoke of syntax", to use van Fraassen's metaphor. The only difference between set-theoretic structures and HOL-structures is that the components of the former are ordered in sequences, whereas the components of the latter are named by symbols. But this difference is not substantial. Thus, the strict semantic view is not a real alternative to the liberal semantic view. They only disagree about whether models should be taken to be set-theoretic structures or rather interpretations of formal languages - but this is no reasonable point of disagreement when the languages in question may be languages of higher-order logic.

In view of this conclusion, let us reassess the main objections against the liberal semantic view and the strong semantic view. The following two sections address the these objections, namely (1) Halvorson's $(2012,2013)$ concern about the strict semantic view that it cannot cope with the problem of equivalence and (2) van Fraassen's (1989, p. 366) concern about the liberal semantic view that the advantages of the semantic view get lost if models are taken to be interpretations of formal languages.

\subsection{The problem of equivalence and the strict semantic view}

The existence of internal higher-order languages of set-theoretic structures implies my conditional claim $\mathrm{C} 2$ : if we find a solution to the problem of equivalence for the liberal semantic view (by making use of syntax), then this solution can be carried over to the strict semantic view. The reason for this is that any given equivalence 
relation $\sim$ between classes of HOL-structures can be indirectly applied to classes of set-theoretic structures by directly applying it to their model-theoretic counterparts. Using the symbol $\sim *$ for indirect $\sim$-equivalence, we can make this idea precise by the following schema.

Definition schema Suppose $\mathbf{C}_{1}$ and $\mathbf{C}_{2}$ are families of set-theoretic structures. $\mathbf{C}_{1} \sim \mathbf{C}_{2}$ iff there are HOL-signatures $\Sigma_{1}$ and $\Sigma_{2}$ fitting $\mathbf{C}_{1}$ and $\mathbf{C}_{2}$ with signature isomorphisms $h_{1}$ and $h_{2}$ such that $\mathbf{C}_{1}^{\Sigma_{1}, h_{1}} \sim \mathbf{C}_{2}^{\Sigma_{2}, h_{2}}$.

The important point here is that $\sim$ may take into account syntax. For instance, it may rely on syntactic notions such as 'formula', 'definition' or 'translation'. This allows to transfer Glymour's (2013) defence of the liberal semantic view against the problem of equivalence to the strict semantic view: the criterion of definitional equivalence for classes of model-theoretic structures can be carried over and applied to classes of set-theoretic structures.

Example Here is a simple example to illustrate how classes of set-theoretic structures can be shown to be definitionally equivalent using internal languages. Topological spaces can be defined in various ways. Sometimes they are defined with open sets as primitive, i.e. in the form $(X, \mathcal{T})$. Sometimes they are defined with interior operators as primitive, i.e. in the form $(X, i n t)$. In practice, these presentation are viewed as equivalent. To justify this one shows how to define a class of open sets in terms of an interior operator and vice versa. Internal languages make it possible to capture this practice in a mathematically rigorous way by demonstrating that the class of topological spaces with open sets as primitive (TOP) is definitionally equivalent ${ }^{6}$ to the class of topological spaces with interior operators as primitive (INT). We consider their canonical HOL-counterparts $\mathbf{T O P}^{\Sigma_{\text {TOP }}}$ and INT ${ }^{\Sigma_{\text {INT }}}$, where $\Sigma_{\mathbf{I N T}}=(\sigma, i)$ is a HOL-signature with the same basic type symbol as $\Sigma_{\text {TOP }}$ and $i$ as a constant of type $\wp(\wp(\sigma) \otimes \wp(\sigma))$. We have to expand the structures on both sides to a common signature $\Sigma^{+}$containing $\sigma$ as basic type and $T$ and $i$ as constants. $\mathbf{T O P}^{\Sigma_{\text {TOP }}}$ can be definitionally expanded to the signature $\Sigma^{+}$using the HOL-formula $\forall_{A \in \wp(\sigma)} i(A)=\bigcup\{U \in T: U \subseteq A\}$. On the other hand, INT ${ }^{\Sigma_{\text {INT }}}$ can be definitionally expanded to the signature $\Sigma^{+}$using the HOL-formula $T=\{A \in \wp(\sigma): A=i(A)\}$. As before, the names of these formulas are written using metalinguistic abbreviation conventions. It follows that these two definitional expansions coincide. Hence, $\mathbf{T O} \mathbf{P}^{\Sigma_{\text {TOP }}}$ and $\mathbf{I N T}^{\Sigma_{\text {INT }}}$ are definitionally equivalent. This justifies calling TOP and INT definitionally equivalent as well (by the definition schema at the beginning of this subsection).

Of course, one could object against Glymour's proposed solution that definitional equivalence is in general not an adequate criterion of equivalence (cf. Barrett and Halvorson 2016, 2017). However, the strategy described above works equally well for more sophisticated criteria such as the criterion of generalised definitional equivalence (also known as 'Morita equivalence') developed by Madarász (2002), Andréka et al. (2008) as well as Barrett and Halvorson (2016), the criterion of bi-interpretability (Ahlbrandt and Ziegler 1986) or the criterion of definable categorical equivalence

6 Classes of structures are definitionally equivalent iff they have a common definitional expansion. For details and a definition of ‘definitional expansion’ see Andréka et al. (2008). 
(Hudetz 2017). These criteria also make use of syntax and have formulations that can be applied to classes of HOL-structures. By means of the above definition schema, they can be applied to set-theoretic structures too.

\subsection{About loss of advantages}

Let us now turn to van Fraassen's worry that we lose some of the advantages of the semantic view if models are taken to be interpretations of formal object languages. Van Fraassen is not specific about what exactly the problem is. However, it seems natural that his worry is that the celebrated language independence of models and theories would be lost if models come with associated languages. Yet, this worry would be in vain. As shown above, set-theoretic structures also come with associated languagesalthough not explicitly. So set-theoretic structures are also not completely language independent. They can only be language independent in the much weaker sense that we do not make use of their associated languages. But we could do the same when it comes to HOL-structures: if we like, we can simply ignore the associated languages of HOL-structures in practice by not making any use of them. Whether and under which circumstances we should or should not make use of formal object languages is a pragmatic and context-dependent question rather than a question of principle. In my opinion, the possibility of explicating intertheoretic relations such as equivalence or reduction by making use of syntactic notions such as definability or translation provides a strong pragmatic reason in favour of using languages at least for some purposes.

So maybe van Fraassen's worry about the liberal semantic view is actually about pragmatic drawbacks. One might fear that using HOL-structures instead of settheoretic structures would be more complicated because it forces us to be unnecessarily explicit about the components of structures when specifying a signature. For example, vector spaces are commonly defined as triples $(V, \oplus, \odot)$ and the underlying field is not understood as a component of the structure. However, in the liberal semantic approach we had to add the field explicitly as a component of the structure. In some situations this might be bothersome and seen as a practical drawback. (Of course, in other situations it could also be a good thing to reinforce exactness.) Be that as it may. The point is that, when using HOL-structures, we do not have to mention all the components in practice if they are clear from the context. We can just as well leave out implicit components in our metalinguistic notation of HOL-structures as we do when dealing with set-theoretic structures. When we talk about vector spaces as triples of the form $(V, \oplus, \odot)$ we silently presuppose that there is some underlying field equipped with the usual operations, although we do not bother mentioning it. Analogously we can silently presuppose that the signature for vector spaces contains a basic type for scalars and symbols for all the usual field operations without writing them down. So using HOL-structures is not more complicated than using set-theoretic structures in this respect.

There is one last concern that needs to be addressed. Van Fraassen (1985) argues that we lose the possibility of characterising important set-theoretic structures (such as the natural number structure and the field of real numbers) when we characterise them 
internally by means of formulas in an object language. However, this objection loses its force when we use higher-order logic with standard semantics. In higher-order logic with standard semantics, we can characterise the natural number structures and field of real numbers uniquely up to isomorphism. Furthermore, the liberal semantic view does not even entail that we should characterise the HOL-structures we are interested in purely internally. We can also characterise them externally if we wish. As noted above, we are not forced to use the languages of HOL-structures for specific purposes. For example, instead of characterising the class of Hausdorff spaces internally by means of formulas of the language $L_{\omega}\left(\Sigma_{\mathbf{T O P}}\right)$, we could also characterise them externally by imposing conditions on them in the metalanguage without making any use of sentences

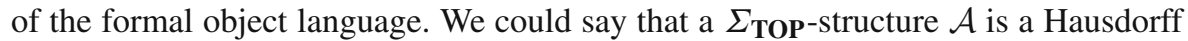
space iff $\emptyset^{\mathcal{A}}$ and $\sigma^{\mathcal{A}}$ are in $T^{\mathcal{A}}, T^{\mathcal{A}}$ is closed under binary intersections, $T^{\mathcal{A}}$ is closed under arbitrary unions and $\left(\sigma^{\mathcal{A}}, T^{\mathcal{A}}\right)$ has the Hausdorff-property. Note that this exactly analogous to the definition of Hausdorff spaces qua set-theoretic structures in the sense of the strict semantic view (see the example at the end of Sect. 3.3). So the presence of object languages is surely not an obstacle for characterising classes of model-theoretic structures. Furthermore, syntactic notions such as satisfaction of formulas or definability are directly applicable even if the classes of structures in question are characterised only externally.

In my opinion, the best way of specifying the class of models of a theory involves both internal and external characterisation. First, one externally characterises a class of HOL-structures serving as a background framework. (For example, these structures may all be required to contain the field of real numbers as a reduct.) In the second step, one lays down the statements specific to the theory. These statements single out the theory's models from the background framework. This approach has the advantage that mathematical assumptions that are not specific to the theory (e.g. real analysis) can be encoded in the background framework and, hence, do not have to be repeated as axioms. Only the specific statements of the theory are used for internal characterisation: they are the only statements in the object language that have to be specified. Thus, they are clearly distinguished from mere framework truths (i.e. sentences that are true in all structures of the background framework). Describing and illustrating this approach in more detail is reserved for future work.

\section{Conclusions}

Several philosophers of science construe models of scientific theories as set-theoretic structures. Some of them go further, claiming that models should not be construed as structures in the sense of model theory. I have argued that if we are ready to construe models as set-theoretic structures (strict semantic view), we could equally well construe them as model-theoretic structures of higher-order logic (liberal semantic and syntactic view). I have shown that every family of set-theoretic structures has an up to signature isomorphism unique model-theoretic counterpart.

The results of this paper support the idea that model-theoretic structures of higherorder logic are able to serve the same purposes as set-theoretic structures. Moreover, model-theoretic structures have the extra advantage of allowing a direct application of 
syntactic concepts such as satisfaction of formulas, definability, translation or definitional equivalence. However, my results also show that-via the counterpart relation mentioned above-it is possible to indirectly apply such syntactic notions to settheoretic structures as well.

Taken together, these results suggest that the dispute between the strict and the liberal semantic view can be overcome. If the strict semantic view on one hand and the liberal semantic view together with the liberal syntactic view on the other hand are the only competing positions left in the syntax-semantics debate (which currently seems to be the case), then the syntax-semantics debate may be regarded as resolved.

Acknowledgements Open access funding provided by Paris Lodron University of Salzburg. I am indebted to Charlotte Werndl for her invaluable support and advice and I thank Sebastian Lutz and Roman Frigg for very helpful feedback and discussions. I also thank two anonymous reviewers for their useful comments.

Open Access This article is distributed under the terms of the Creative Commons Attribution 4.0 International License (http://creativecommons.org/licenses/by/4.0/), which permits unrestricted use, distribution, and reproduction in any medium, provided you give appropriate credit to the original author(s) and the source, provide a link to the Creative Commons license, and indicate if changes were made.

\section{References}

Ahlbrandt, G., \& Ziegler, M. (1986). Quasi-finitely axiomatizable totally categorical theories. Annals of Pure and Applied Logic, 30, 63-83.

Andréka, H., Madarász, J., \& Németi, I. (2008). Defining new universes in many-sorted logic (manuscript). Awodey, S. (1997). Logic in Topoi: Functorial semantics for higher-order logic. Ph.D. Dissertation, Chicago. Balzer, W., Moulines, U., \& Sneed, J. (1987). An architectonic for science: The structuralist program. Dordrecht: Springer.

Barrett, T., \& Halvorson, H. (2016). Morita equivalence. The Review of Symbolic Logic, 9(3), 556-582.

Barrett, T., \& Halvorson, H. (2017). From geometry to conceptual relativity. Erkenntnis. https://doi.org/10. 1007/s10670-016-9858-y.

Bourbaki, N. (1968). Theory of sets. Paris: Hermann.

Carnap, R. (1958). Beobachtungssprache und theoretische Sprache. Dialectica, 12(3/4), 236-248.

Da Costa, N., \& French, S. (2003). Science and partial truth: A unitary approach to models and scientic reasoning. New York: Oxford University Press.

De Bouvere, K. (1965). Synonymous theories. In J. Atkinson, L. Henkin, \& A. Tarski (Eds.), The theory of models. Amsterdam: North Holland.

Diaconescu, R. (2008). Institution-independent model theory. Basel: Birkhäuser.

French, S., \& Ladyman, J. (1999). Reinflating the semantic approach. International Studies in the Philosophy of Science, 13(2), 103-121.

Glymour, C. (2013). Theoretical equivalence and the semantic view of theories. Philosophy of Science, 80(2), 286-297.

Goguen, J., \& Burstall, R. (1992). Institutions: Abstract model theory for specification and programming. Journal of the Association for Computing Machinery, 39(1), 95-146.

Halvorson, H. (2012). What scientific theories could not be. Philosophy of Science, 79(2), 183-206.

Halvorson, H. (2013). The semantic view, if plausible, is syntactic. Philosophy of Science, 80(2), 475-478.

Hendry, R. F., \& Psillos, S. (2007). How to do things with theories: An interactive view of language and models in science. In J. Brzeziski, A. Klawiter, T. A. F. Kuipers, K. Lastowski, K. Paprzycka, \& P. Przybysz (Eds.), The courage of doing philosophy: Essays dedicated to Leszek Nowak (pp. 123-157). Amsterdam: Rodopi.

Henkin, L. (1950). Completeness in the theory of types. Journal of Symbolic Logic, 15(2), 81-91.

Hudetz, L. (2017). Definable categorical equivalence (manuscript). https://www.academia.edu/29993937/. Johnstone, P. (2002). Sketches of an elephant: A topos theory compendium (Vol. I, II). Oxford: Oxford University Press. 
Lambek, J., \& Scott, P. (1986). Introduction to higher order categorical logic. Cambridge, MA: Cambridge University Press.

Lutz, S. (2014a). the semantics of scientific theories. In A. Brozek, J. Jadacki (Eds.) Ksiega pamiatkowa Marianowi Przełeckiemu w darze na 90-lecie urodzin (pp. 33-67). Norbertinum

Lutz, S. (2014b). What's right with a syntactic approach to theories and models? Erkenntnis, 79, 1475-1492.

Lutz, S. (2015). What was the syntax-semantics debate in the philosophy of science about? Philosophy and Phenomenological Research. https://doi.org/10.1111/phpr.12221.

Madarász, J. (2002). Logic and relativity (in the light of definability theory). Ph.D. Dissertation.

Orey, S. (1959). Model theory for the higher order predicate calculus. Transactions of the American Mathematical Society, 92, 72-84.

Przełecki, M. (1969). The logic of empirical theories. London: Routledge and Kegan Paul.

Scheibe, E. (1997). Die Reduktion physikalischer Theorien. Ein Beitrag zur Einheit der Physik Teil I: Grundlagen und elementare Theorie. Berlin: Springer.

Tarski, A., Mostowski, A., \& Robinson, R. M. (1953). Undecidable theories. Amsterdam: North-Holland Publishing Company.

van Fraassen, B. (1985). Empiricism in the philosophy of science. In P. M. Churchland \& C. A. Hooker (Eds.), Images of science: Essays on realism and empiricism, with a reply from Bas C. van Fraassen (pp. 245-308). Chicago: University of Chicago Press.

van Fraassen, B. C. (1989). Laws and symmetry. Oxford: Oxford University Press.

van Fraassen, B. C. (2014). One or two gentle remarks about Hans Halvorson's critique of the semantic view. Philosophy of Science, 81(2), 276-283.

von Neumann, J. (1937). Über ein ökonomisches gleichungssystem und ein verallgemeinerung des brouwerschen fixpunktsatzes. Ergebnisse eines Mathematischen Kolloquiums, 8, 73-83.

Worrall, J. (1984). An unreal image. British Journal for the Philosophy of Science, 35(1), 65-80. 